\title{
Solar luminance distribution in the principal plane for different wavelengths at two locations in Sri Lanka
}

\author{
CP Abayaratne ${ }^{1^{*}}$, AVUA Wickramarathna ${ }^{1^{*}}$ and MK Jayananda ${ }^{2^{*}}$ \\ ${ }^{1}$ Department of Physics, Faculty of Applied Sciences, University of Sri Jayewardenepura, Nugegoda. \\ ${ }^{2}$ Department of Physics, Faculty of Science, University of Colombo, Colombo 3.
}

\begin{abstract}
The colour of the sky has been the subject of many studies related to solar luminance distribution. This paper presents the angular sky luminance distributions measured at the ground level for white light and three spectral bands centred at blue $(450 \mathrm{~nm})$, green $(550 \mathrm{~nm})$ and red $(650 \mathrm{~nm})$ in the principal plane of the sun at two locations in Sri Lanka. The luminance measurements were taken by an LDR detector along with three colour filters. Results reveal that the groundlevel angular luminance distributions of white, green, and red light are similar with high peak levels of direct sun luminance which sharply decrease away from the solar disc followed by a relatively small gradual rise for angular solar distances exceeding $90^{\circ}$. In contrast, the direct sun luminance for blue light shows a small peak directly under the sun which too decreases away from the solar disc and remains with little variation up to $90^{\circ}$ but rises to comparatively high levels of luminance for angular solar distances above $90^{\circ}$. The measured blue peak luminance under direct sunlight is higher in Kandy which is at a higher altitude with a shorter air column above the site compared to Mahiyanganaya where the peak luminance is smaller due to more light being scattered by the longer air column at the lower altitude in Mahiyanganaya.
\end{abstract}

Keywords: Atmospheric scattering, sky luminance, skylight scattering, solar radiance distribution.

\section{INTRODUCTION}

The colour of the sky, which depends on the spectral composition, has been of interest to many scientists and laymen for centuries. According to the famous Rayleigh theory of scattering of solar radiation by atmospheric air molecules, the extent of scattering is inversely proportional to the fourth power of the wavelength and explains the blue colour of the sky (Coulson, 1988; Bohren, 2007; Mani, 2008). Any deviations to the composition of the atmosphere such as the presence of anisotropic molecules, aerosols, their sizes and surface albedo can cause measurable differences in the spectral intensity distribution across the sky, although it may not be noticeable to the naked eye. Details of atmospheric conditions such as concentration and size distribution of aerosols can be determined with the use of solar intensity measurements (Rangarajan \& Mani, 1984; Kaskaoutis \& Kambezidis, 2006; Rosairo et al., 2011; Djafer \& Irbah, 2013). Scattering by particles with dimensions comparable to or larger than the wavelength of the visible light is described by Mie scattering, which does not depend on the wavelength but strongly depends on conditions such as air pollution and cloudiness (Wald, 2018). Clouds appear in white because of Mie scattering by water droplets which are large compared to the wavelengths of the visible light (Wald, 2018). In contrast to Rayleigh scattering, Mie scattering is more complicated and it has a strong forward scattering where the diameter of the particles is the same or larger than the incident wavelength. Mostly, dust particles have size distributions comparable with the wavelength of the visible light, water droplets, ice crystals and

\footnotetext{
* Corresponding author (cmapala@sjp.ac.lk (iD https: //orcid.org/ : 0000-0001-6762-6444)
} 
aerosols. When the particle diameter is much larger than the wavelength of visible light, a theoretically more complex type of atmospheric scattering independent of the wavelength known as non selective scattering explains the haze seen in the lower atmosphere caused by water droplets and large aerosols (Wald, 2018). All these factors as well as ground reflections contribute to the spectral composition of skylight.

Visible spectrum together with near ultraviolet and near infrared radiation attributes to $99 \%$ solar radiation while the visible region attributes to $40 \%$ of the solar radiation received at the earth's surface on clear days (Mani, 2008). The amount of short wavelength solar radiation that reaches the earth's surface is limited due to absorption by oxygen and ozone at high altitudes of the atmosphere, and spectral distribution of solar radiation gives a peak around $550 \mathrm{~nm}$ wavelength at the mean sea level (Mani, 2008). Although many scientific articles about the spectral intensity distribution of sunlight along with images are available in the literature, not many such articles can be found on the angular distribution of skylight which is sunlight scattered by the constituents of the atmosphere. Some measured relative skylight intensity distributions carried out in several locations in USA using radiometers based on photomultipliers have been presented by Coulson (1988). Contour plots of measured radiance distribution using a CCD camera system over certain regions in USA have been reported by Liu and Voss (1997). Hisdal (1986) gives spectral irradiance distributions under different sky conditions. However, such measurements have not been carried out in Sri Lanka and the purpose of this work is to present the angular distribution of sky luminance for three spectral bands centred at 450, 550 and $650 \mathrm{~nm}$. The angular distribution of sky luminance found would be of interest to better understand the processes that take place in the atmosphere through the interaction of direct and scattered sunlight with regional atmospheric constituents. For easy reference, the formula for the intensity $I_{R}$ for the light of wavelength $\lambda$ scattered by an angle $\phi$ in Rayleigh scattering is given in Equation 1, where $\varepsilon$ and $\mathrm{n}$ are the relative permittivity and the refractive index of the droplets, respectively, while $\varepsilon_{0}$ and $\mathrm{n}_{0}$ are those of the surrounding medium (Wald, 2018).

$$
I_{R}=\frac{1}{2}\left(\frac{2 \pi n_{0}}{\lambda}\right)^{4} r^{6}\left[\frac{\left(\varepsilon-\varepsilon_{0}\right)}{\left(\varepsilon+2 \varepsilon_{0}\right)}\right]^{2}\left(1+\cos ^{2} \phi\right)
$$

The length of the direct geometrical pathway (known as relative air mass) through the atmosphere affects the attenuation of the solar radiation, and is given by the formula of Equation 2 (Luo, 2016);

$$
\text { Air mass }=\frac{1}{\sin \theta}\left[\frac{P}{P_{0}}\right]
$$

where $\theta$ is the solar elevation angle, $\mathrm{P}$ is the pressure at a given altitude (in $\mathrm{Pa}$ ) and $\mathrm{P}_{0}$ is the pressure at sea level (101325 Pa). If the direct path way is shorter as during midday, attenuation is less and if the path way is longer as at sunrise and sunset, more attenuation can take place. The main focus of this paper is to present the observations with possible implications that can be extended into the UV region, based on the results of angular spectral distribution of the sky luminance on the principal plane of the sun, for some solar elevation angles during the morning hours between 0700-0930 h. A brief description of the instrument used for measurements and the procedure followed are given in the Methodology section. Results are presented in the following section with a qualitative discussion and some implications of the results to be continued in a future investigation.

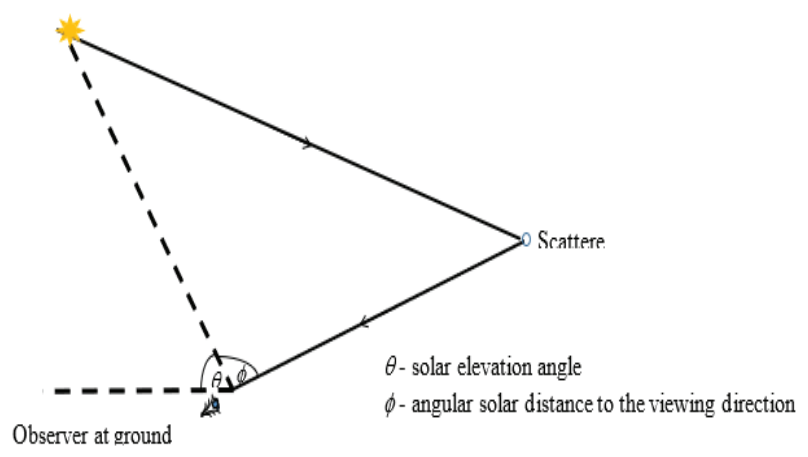

Figure 1 : Solar elevation angle $\theta$ and the angular solar distance $\varnothing$ to viewing direction in the principal plane

The principal plane carries more information than any other plane across the sky (Coulson, 1988; Liu \& Voss, 1997; Dahlberg et al., 2011). The vertical plane passing through the sun, a scatter particle and an observer is known as the principal plane of the sun and is illustrated in Figure 1 when the Sun is at a solar elevation angle $\theta$. Figure 1 also shows a light ray scattered towards the eye of an observer at the ground level having an angular distance $\phi$ with the sun. Measurements were taken using an LDR detector and were later calibrated to find the sky luminance in Lux.

\section{METHODOLOGY}

Luminance measurements at the ground level were obtained using a fully automated polarimeter (Figure 2) constructed to find the angular distribution of the skylight polarization in the principal plane. This 
polarimeter is an improvement over a partially automated polarimeter constructed earlier (Abayaratne et al., 2016)

A window comparator unit was used as a suntracking unit and three hybrid motors were used for movements of the polarimeter. To observe the temperature and humidity there are inbuilt humidity and temperature sensors (DHT 11). The LDR was placed at the bottom of a PVC tube with one end of the tube fitted with an end-cap having a small aperture $(\sim 1 \mathrm{~cm})$ in the centre to use as a collimator, allowing a narrow beam of light to fall on the detector with an acceptance angle of $2^{\circ}$. Three optical filters of centre wavelengths of $450 \mathrm{~nm}$, $550 \mathrm{~nm}$, and $650 \mathrm{~nm}$ and each of half power bandwidth $\pm 40 \mathrm{~nm}$ was placed on the aperture consecutively to select the appropriate wavelength band or to allow white light to enter through the collimator. Each filter had a transmissivity of $60 \%$, for which a correction was made for the luminance measurements.

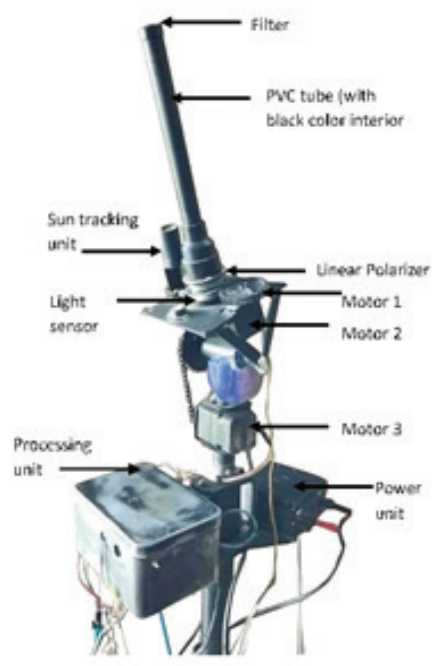

Figure 2: Fully automated polarimeter

A polarizer was placed between the aperture and the detector, since the instrument was initially constructed to find the degree of polarization, which is not required in measuring the total sky luminance.
First, the instrument tracks the sun through rotations around a horizontal axis and a vertical axis and records the position of the sun. At this point, the collimator of the instrument scans the principal plane when it rotates around the horizontal axis. Next, it goes back to the starting point (horizontal direction in the principal plane of the sun) and moves two degrees at a time in the principal plane of the sun and at each such point rotates the polarizer in three degree steps 60 times and reads the corresponding voltage across the LDR sensor. After that, it chooses the maximum and minimum values of the voltage readings in two mutually perpendicular directions. These voltages were later converted to luminance (in Lux) after calibrating with the Lux sensor (BH1750FVI) and polarizers to control the intensity of light emitted by an LED source.

Due to limitations of the source intensity, the luminance received by the detector was limited to 1000 Lux. Therefore, measured intensities exceeding 1000 Lux were obtained by extrapolating the calibration curve, which imposed a limitation on the accuracy of intensities above 1000 Lux. Furthermore, intensities of blue and red light were much lower compared to the green light due to the lower source emissions in blue and red spectral regions and lower detector sensitivity, which makes it difficult to calibrate the voltages with red and blue light. Due to this reason, the spectral dependence of the detector response was not taken into consideration. After reading the required voltages, the collimator rotates by two degrees automatically as programmed by an Arduino platform until the entire principal plane is scanned.

The luminance values corresponding to the two polarizations, $\mathrm{I}_{\max }$ and $\mathrm{I}_{\min }$ can be added to obtain the total intensity $\mathrm{I}(\theta)$. $\left(\mathrm{I}(\theta)=\mathrm{I}_{\max }+\mathrm{I}_{\min }\right)$. Measurements were taken in two locations in Sri Lanka, Kandy and Mahiyanganaya. As far as the content of this paper is concerned, the difference between the two sites is the altitude difference. Geographical details of the locations and the dates of data collection are given in Table 1.

Table 1: Geographical details of the measurement sites and the dates of data collection

\begin{tabular}{lcccc}
\hline \multicolumn{1}{c}{ Location } & Latitude & Longitude & Altitude & Data collection dates \\
\hline Kandy & $7.29 \mathrm{~N}$ & $80.63 \mathrm{E}$ & $586 \mathrm{~m}$ & $01,02,03,04,05$ May 2018 \\
Mahiyanganaya & $7.33 \mathrm{~N}$ & $80.99 \mathrm{E}$ & $90 \mathrm{~m}$ & $20,21,22,23$ July 2018 \\
\hline
\end{tabular}




\section{RESULTS AND DISCUSSION}

Figures 3(a) and (b) show two images taken on 01.05.2018 at $0750 \mathrm{~h}$ of the East and West sky respectively, in Kandy. Figures 4 (a), 4(c), 4(e) and 4(g) represent the variation of the average solar luminance (measured in Lux) over 4 days around the same time (nearly the same solar elevation angle $\theta$ ) at the ground level as a function of $\phi$ on the principal plane in Kandy for white (without filters), blue, green and red light respectively while Figures 4 (b), 4(d), 4(f) and 4(h) represent the same for Mahiyanganaya. Each data set was collected within a period of about 8 minutes. The respective mean time and mean solar elevation angle for each data set is given in the inset of each figure.

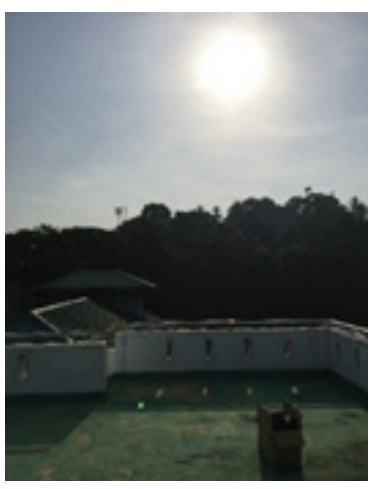

(a)

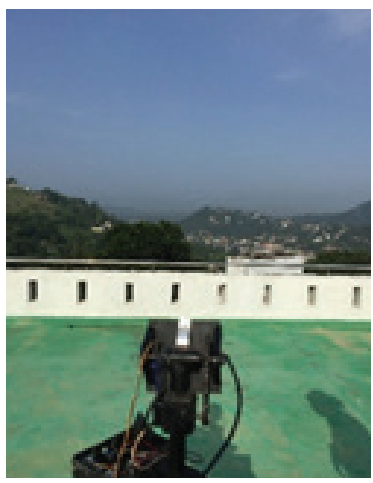

(b)

Figure 3: Images of the (a) East and (b) West sky in Kandy taken on 01.05 .2018 around $0750 \mathrm{~h}$.

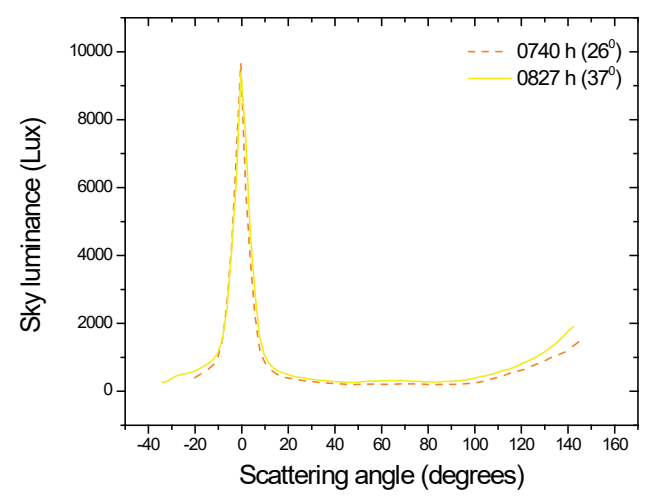

(a): Kandy - White light

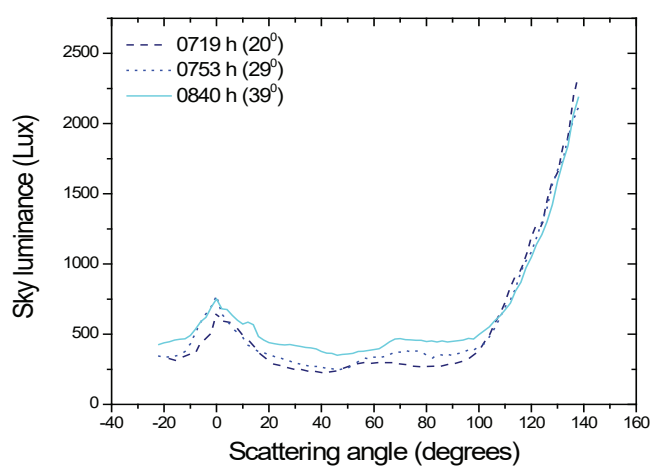

(c): Kandy - Blue light (450 nm)

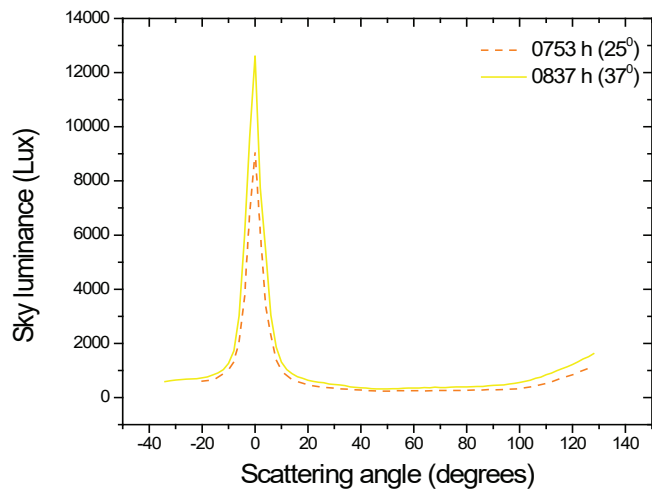

(b): Mahiyanganaya - White light

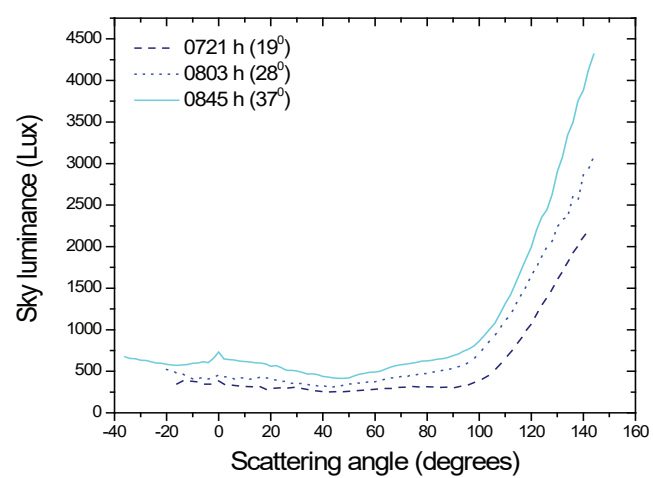

(d): Mahiyanganaya - Blue light (450 nm) 


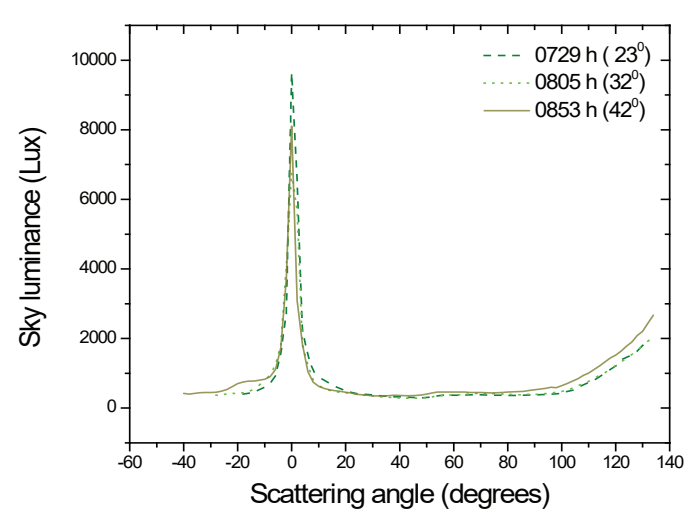

(e): Kandy - Green light (550 nm)

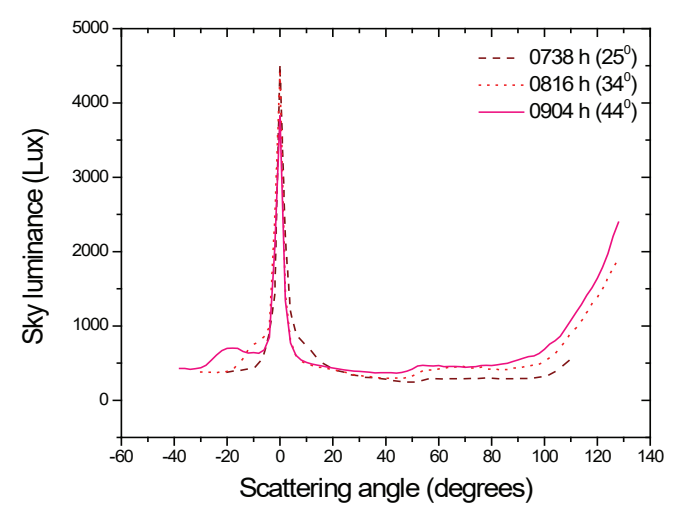

(g): Kandy - Red light (650 nm)

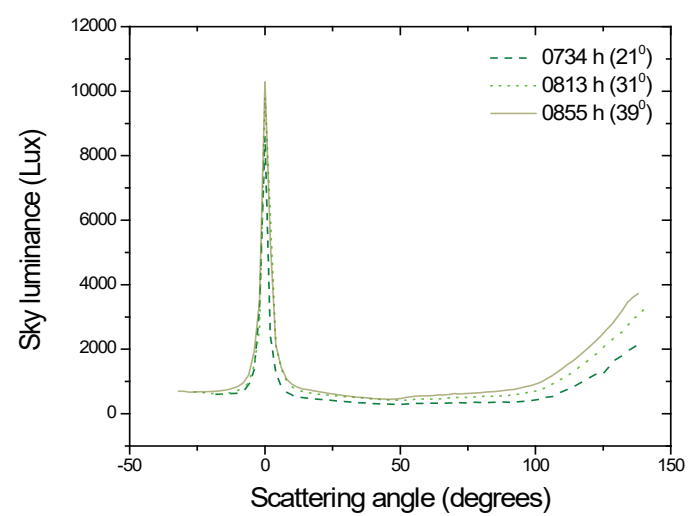

(f): Mahiyanganays - Green light $(550 \mathrm{~nm})$

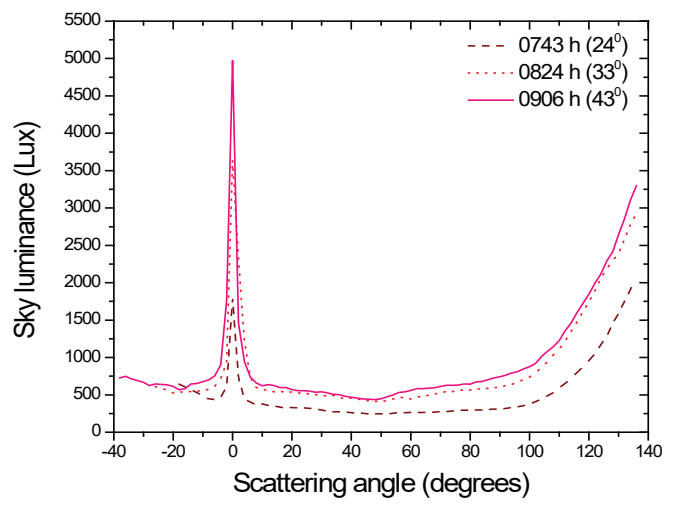

(h): Mahiyanganaya - Red light (650 nm)

Figure 4: Variations of solar luminance with angular solar distance at the mean times and solar elevation angles given in the inset for white light and the three different wavelengths for Kandy (a, c, e, g) and Mahiyanganaya (b, d, f, h)

Some results expected from theory can be verified using the graphs of Figure 4 . It can be easily seen by comparing the three peaks in each graph of Figure 4 separately for each location that the direct sun luminance increases with increasing solar elevation angle since the path travelled through the atmosphere decreases with the rising sun (and decreasing relative air mass), in accordance with the expected result. (It should be noted that there was a time gap of about 10 minutes and a difference of about $10^{\circ}$ in the solar elevation angle between measurements for each graph in the order white, blue, green and red.)

Moving away from the sun through the principal plane, the solar intensity gradually decreases until the angular distance is about $6^{\circ}$, and remains without significant variation until the angular solar distance $\phi$ reaches about $90^{\circ}$. For values of $\phi$ above $90^{\circ}$, all graphs show a gradual increase in the solar luminance. Out of all these graphs those for blue light are of particular interest, which is the most important finding of this work. While the graphs for white, green and red light look quite similar in the angular distribution of solar luminance in the principal plane, all graphs for blue light $(450 \mathrm{~nm})$ show higher levels of luminance for angular solar distances exceeding $100^{\circ}$ relative to the direct Sun luminance. This is qualitatively explained by the higher scattering undergone by shorter wavelengths as predicted by Rayleigh theory. 
Table 2: Direct solar luminance, angular solar distance when the scattered light intensity is equal to the direct solar radiance and the sky luminance at an angular solar distance of $135^{\circ}$ for blue light

\begin{tabular}{cccccc}
\hline Location & $\begin{array}{c}\text { Solar } \\
\text { elevation } \\
\text { angle }(\phi)\end{array}$ & Time & $\begin{array}{c}\text { Direct solar } \\
\text { luminance } \\
(\text { Lux })\end{array}$ & $\begin{array}{c}\text { Angular solar distance }(\theta) \text { to a } \\
\text { direction with luminance equal to } \\
\text { that directly under the Sun }\end{array}$ & $\begin{array}{c}\text { Luminance at an angular solar } \\
\text { distance of } 135^{\circ} \text { (Lux) }\end{array}$ \\
\hline Kandy & $20^{\circ}$ & $0719 \mathrm{~h}$ & 652 & $108^{\circ}$ & 2047 \\
& $29^{\circ}$ & $0753 \mathrm{~h}$ & 751 & $113^{\circ}$ & 1964 \\
& $39^{\circ}$ & $0840 \mathrm{~h}$ & 751 & $114^{\circ}$ & 1978 \\
& $19^{\circ}$ & $0721 \mathrm{~h}$ & 385 & $100^{\circ}$ & 2489 \\
& $28^{\circ}$ & $0803 \mathrm{~h}$ & 456 & $94^{\circ}$ & 3420 \\
\hline
\end{tabular}

Despite the sky being overcast with a cloud cover during the morning hours when data was collected in Kandy after rain the previous night on each of the four days compared to Mahiyanganaya, it can be seen that the direct solar radiance at nearly equal solar elevation angles (approximately $20^{\circ}, 28^{\circ}, 38^{\circ}$ ) are higher for blue light in Kandy where the air column above the ground level is shorter than that in Mahiyanganaya due to the higher altitude of the Kandy site while the scattered light seen at an angular solar distance of $135^{\circ}$ is higher in Mahiyanganaya. As can be expected from Rayleigh theory of scattering that blue light is scattered more in Mahiyanganaya during the longer travel path through the atmosphere thus displaying a reduced direct solar luminance and a higher sky luminance for higher angular solar distances. In a similar manner, the scattered skylight luminance can be observed to reach a value equal to the direct solar luminance sooner (at a lower value of $\phi$ ) in Mahiyanganaya compared to Kandy. (Note also that this is not observed for the other two colours or white light possibly due to the different atmospheric conditions.)

The observation of higher luminances in directions away from the sun for blue light can be expected from UV light as well due to the high degree of scattering of UV light according to the Rayleigh Theory. Although the luminance directly under the sun is low for shorter wavelengths such as those of UV light, a high luminance can be expected in the backscattered light reaching an observer from the direction opposite to the sun. In order to prevent being exposed to UVA radiation during the morning hours, we may need to shield ourselves from solar radiation reaching us from angular solar distances above $100^{\circ}$ rather than from the direct sun. In other words, the radiation coming from directions opposite to the sun carries more of blue (and possibly also UVA light) than the light coming directly from the sun at solar elevation angles below $40^{\circ}$ considered in this work. As a future extension of this work, this fact can be investigated using a suitable UV sensor to measure UV intensity. This would be of importance for Sri Lanka which is a tropical country with high values of UV index and no reported investigations.

Health hazards such as skin cancers and cataract in the eyes caused by exposure to UV radiation, have been published by the World Health Organization (WHO, 2020). The intensity of UV radiation is indicated by the UV Index, which is the cumulative effect of UV radiation coming from all directions (Vanicek et al., 2000). When measuring the UV index, the usual practice is to place the UV sensor horizontally and measure the UV radiation incident on the sensor from all directions (Koepke et al., 1998). Scanning the principal plane could be used as an alternative method which also provides additional information such as the angular distribution of UV radiation.

Figure 5 shows the angular luminance distributions obtained by averaging the data taken on four days in each location for solar elevation angles of approximately $40^{\circ}$ for white light, blue, green and red bands studied here in the same figure for Kandy and Mahiyanganaya indicating the decreasing levels of luminance in the order green, red and blue as expected. However it is noteworthy that the luminance values in Figures 4 and 5 have limited accuracy especially those exceeding 1000 Lux as a result of calibration errors and disregarding the exact spectral dependence of the LDR. It would be possible to improve the accuracy if the LDR sensor is replaced with a Lux sensor and the spectral dependence of the sensor is taken into account. However, the main conclusions of the paper are not affected by this inaccuracy.

Throughout the periods that data was collected, a fair amount of clouds were present in the sky in both sites which could be the reason for the irregularities 

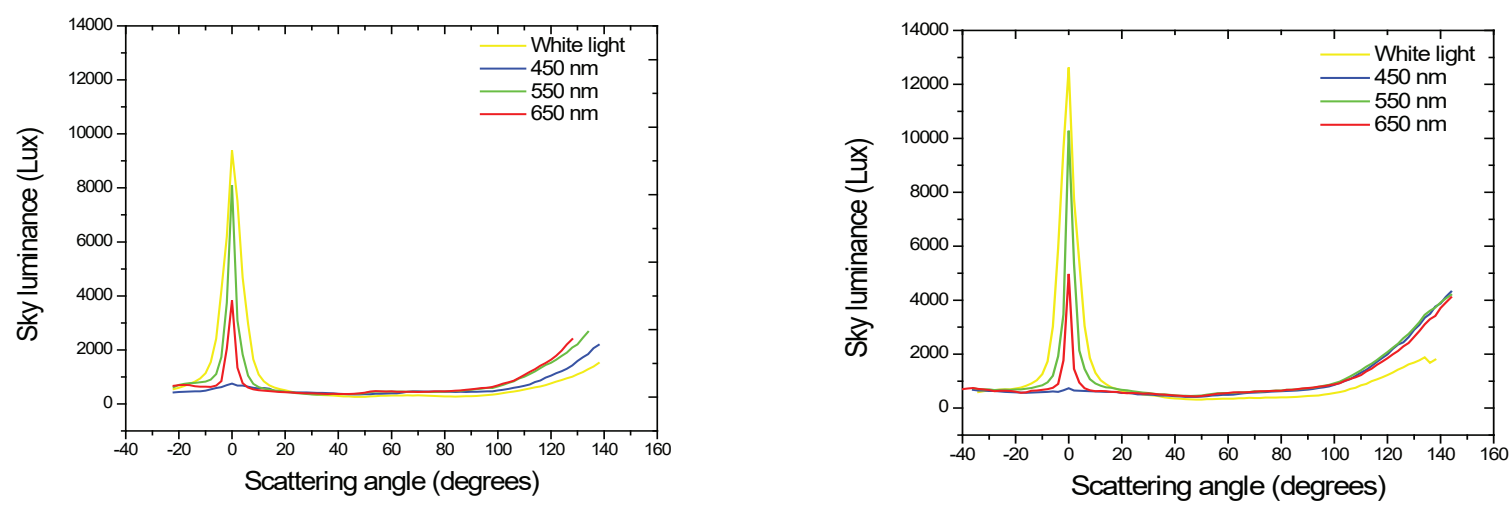

Figure 5: Variation of sky luminance with angular solar distance for different wavelengths given in the inset at approximately $40^{\circ} \pm 10^{\circ}$ at (a) Kandy (b) Mahiyanganaya

observed in all graphs. According to the cloud cover data obtained from the Climate Division, Department of Meteorology, Sri Lanka, on 04.07.2019, in Katugastota (Kandy) and Badulla (near Mahiyanganaya), given in Table 3, a crude average taken for the relevant data indicates thicker cloud cover in Kandy. The temperatures observed in Kandy ranged from $24{ }^{\circ} \mathrm{C}-32{ }^{\circ} \mathrm{C}$, while the humidity varied between $43 \%-80 \%$. The temperatures in Mahiyanganaya were between $28{ }^{\circ} \mathrm{C}-40{ }^{\circ} \mathrm{C}$ and the

Table 3: Cloud cover data obtained from the Climate Division, Department of Meteorology, Sri Lanka on 04.07.2019.

(Higher values indicate thicker cloud covers)

\begin{tabular}{lccc}
\hline \multicolumn{2}{c}{ Katugastota, Kandy } & \multicolumn{2}{c}{ Badulla } \\
Date & $\begin{array}{c}\text { Cloud } \\
\text { cover }\end{array}$ & Date & $\begin{array}{c}\text { Cloud } \\
\text { cover }\end{array}$ \\
\hline 01.05 .2018 & 4 & 20.07 .2018 & 3 \\
02.05 .2018 & 4 & 21.07 .2018 & 4 \\
03.05 .2018 & 4 & 22.07 .2018 & 2 \\
05.05 .2018 & 3 & 23.07 .2018 & 3 \\
Average & 3.75 & & 3 \\
\hline
\end{tabular}

\section{CONCLUSION}

Angular sky luminance distribution in the principal plane of the sun obtained using sky luminance measurements made with the use of an LDR sensor and an observation hitherto not reported in the literature to the best of our knowledge has been presented in this paper. Graphs illustrate that the peak luminance increases with the solar elevation angle $\theta$ for all colour bands, blue, green and red as well as white light in agreement with our common experience. The key finding of this work, which can be expected from the Rayleigh theory of scattering but often overlooked in practice, is the presence of more intense blue light in the sky opposite the sun compared to the direct solar luminance. The directional spectral dependence of the brightness of the sky could be useful in understanding the scattering processes in the atmosphere further and to extract details of the atmospheric constituents. These observations made with a low cost instrument can be refined using more sophisticated instruments. As a further extension of this work, the experiment could be performed with a UV sensor to determine the angular intensity distribution of UV light to check whether significant UV radiation arrives from directions other than that of the Sun.

\section{Acknowledgements}

The authors thank the University of Sri Jayewardenepura for providing funds through the university grant ASP/06/ RE/SCI/2013/016. The authors also acknowledge Prof. D.A. Tantrigoda for his advice..

\section{REFERENCES}

Abayaratne C.P., Wickramarathna A.V.U.A., Rodrigo D.D. \& Mannathunga K.S. (2016). A low-cost partially automated polarimeter for investigating skylight polarization. Journal of the National Science Foundation of Sri Lanka 44(1): 95-103.

DOI: http://dx.doi.org/10.4038/jnsfsr.v44i1.7986

Bohren C.F. (2007). Atmospheric optics. In: The Optics Encyclopaedia: Basic Foundations and Practical Applications (eds. T.G. Brown, K. Creath, H. Kogelnik, M.A. Kriss, J. Schmit \& M.J. Weber). Wiley-VCH Verlag GmbH \& Co., Germany.

DOI: https://doi.org/10.1002/9783527600441.oe004 
Coulson K.L. (1988). Polarization and Intensity of Light in the Atmosphere, pp. 375-391. A. Deepak Publishers. Hampton, USA.

Dahlberg A.R., Pust N.J. \& Shaw J.A. (2011). Effects of surface reflectance on skylight polarization measurements at the Mauna Loa Observatory. Optics Express 19(17): 16008-16021.

do Rosário N.E., Yamasoe M.A. \& Longo K.M. (2009). Aerosol optical depth and ångström coefficient retrievals over the Amazon forest during 2007 biomass burning season. AIP Conference Proceedings, American Institute of Physics 1100(01): 494-497.

Djafer D. \& Irbah A. (2013). Estimation of atmospheric turbidity over Ghardaïa city. Atmospheric Research 128: 76-84.

DOI: https://doi.org/10.1016/j.atmosres.2013.03.009

Hisdal V. (1986). Spectral distribution of global and diffuse solar radiation in Ny-Alesund, Spitsbergen. Polar Research 5: 1-27.

Kaskaoutis D.G. \& Kambezidis H.D. (2006). Investigation into the wavelength dependence of the aerosol optical depth in the Athens area. Quarterly Journal of the Royal Meteorological Society: A Journal of the Atmospheric Sciences, Applied Meteorology and Physical Oceanography 132(620): 2217-2234.

DOI: https://doi.org/10.1256/qj.05.183

Koepke P. et al. (24 authors) (1998). Comparison of models used for UV index calculations. Photochemistry and Photobiology 67(6): 657-662.

Liu Y. \& Voss K. (1997). Polarized radiance distribution measurement of skylight. II. Experiment and data. Applied Optics 36(33): 8753-8764.

Luo R. (ed.) (2016). Encyclopedia of Color Science and Technology. Springer-Verlag, New York, USA.

Mani A. (2008). Handbook of Solar Radiation Data for India. Resonance November 2008: 1082-1086. Available at https://www.ias.ac.in/article/fulltext/reso/013/11/10821086

Rangarajan S. \& Mani A. (1984). A new method for the determination of atmospheric turbidity. Tellus B: Chemical and Physical Meteorology 36(1): 50-54. DOI: https://doi.org/10.3402/tellusb.v36i1.14804

Vanicek K., Frei T., Litynska Z. \& Schmalwiese A. (2000). UVIndex for the Public.Aguide for publication and interpretation of solar UV Index forecasts for the public prepared by the Working Group 4 of the COST-713 Action 'UVB Forecasting'. European Communities, Brussels, Belgium.

Wald L. (2018). Basics in solar radiation at Earth surface. Available at https://hal-mines-paristech.archives-ouvertes. fr/hal-01676634

WHO (2020). Ultraviolet radiation and health, Available at https://www.who.int/uv/uv_and_health/en/, accessed on 16 October 2020. 